OPEN ACCESS

Edited by:

Chunfu Zheng,

University of Calgary, Canada

Reviewed by:

Iffan Ullah,

Karakoram International University,

Pakistan

Nathaniel Sands,

University of Pennsylvania,

United States

*Correspondence:

Shun Li

lishun@cmc.edu.cn

Hai Liao

ddliaohai@home.swjtu.edu.cn

Specialty section:

This article was submitted to

Virology,

a section of the journal

Frontiers in Microbiology

Received: 31 December 2021

Accepted: 19 January 2022

Published: 02 March 2022

Citation:

Liang H, Luo D, Liao H and Li S (2022) Coronavirus Usurps the Autophagy-Lysosome Pathway and Induces Membranes Rearrangement for Infection and Pathogenesis.

Front. Microbiol. 13:846543 doi: 10.3389/fmicb.2022.846543

\section{Coronavirus Usurps the Autophagy-Lysosome Pathway and Induces Membranes Rearrangement for Infection and Pathogenesis}

\author{
Haowei Liang ${ }^{1,2}$, Dan Luo ${ }^{1}$, Hai Liao ${ }^{2 *}$ and Shun $\mathrm{Li}^{1,3 *}$ \\ 1 Department of Immunology, School of Basic Medical Sciences, Chengdu Medical College, Chengdu, China, ${ }^{2}$ School \\ of Life Sciences and Engineering, Southwest Jiaotong University, Chengdu, China, ${ }^{3}$ Non-coding RNA and Drug Discovery \\ Key Laboratory of Sichuan Province, Chengdu Medical College, Chengdu, China
}

Autophagy is a crucial and conserved homeostatic mechanism for early defense against viral infections. Recent studies indicate that coronaviruses (CoVs) have evolved various strategies to evade the autophagy-lysosome pathway. In this minireview, we describe the source of double-membrane vesicles during CoV infection, which creates a microenvironment that promotes viral RNA replication and virion synthesis and protects the viral genome from detection by the host. Firstly, CoVs hijack autophagy initiation through non-structural proteins and open-reading frames, leading to the use of non-nucleated phagophores and omegasomes for autophagy-derived double-membrane vesicles. Contrastingly, membrane rearrangement by hijacking ERassociated degradation machinery to form ER-derived double-membrane vesicles independent from the typical autophagy process is another important routine for the production of double-membrane vesicles. Furthermore, we summarize the molecular mechanisms by which CoV non-structural proteins and open-reading frames are used to intercept autophagic flux and thereby evade host clearance and innate immunity. A comprehensive understanding of the above mechanisms may contribute to developing novel therapies and clinical drugs against coronavirus disease 2019 (COVID-19) in the future.

\footnotetext{
Keywords: coronavirus, double membrane vesicles (DMV), membranes rearrangement, virus escape, autophagylysosome pathway
}

\footnotetext{
Abbreviations: ATG, autophagy-related proteins; CQ, chloroquine; CoV, coronavirus; EDEM1, ER degradation enhancing alpha-mannosidase-like protein-1; DMV, double-membrane vesicles; ER, endoplasmic reticulum; IFN-I, type I interferon; IRF3, interferon regulatory factor-3; MERS, the Middle East respiratory syndrome; NSP, non-structural proteins; ORF, openreading frame; SARS, severe acute respiratory syndrome; STING, stimulator of interferon gene; TLR, toll-like receptors; TBK1, TANK binding kinase 1; TRIF, TIR domain-containing adaptor inducing interferon- $\beta$.
} 


\section{INTRODUCTION}

At the beginning of the 21st century, we have witnessed the outbreak of three types of diseases by coronavirus (CoV) infection, which are severe acute respiratory syndrome (SARS), Middle East respiratory syndrome (MERS), and coronavirus disease 2019 (COVID-19). CoVs have caused serious public health problems and economic losses. Although various countries have begun to vaccinate against SARS-CoV-2, there is still an urgent need to find effective antiviral drugs to treat COVID-19 (de Wit et al., 2020). Therefore, it is crucial to discover new drug targets according to viral replication and infection in host cells.

Coronaviruses (CoVs) are enveloped viruses with a positivesense, single-stranded RNA (ssRNA) genome. Although the specific process of $\mathrm{CoV}$ infection of host cells is not yet completely clear, according to previous studies, the infection cycle of CoVs is divided into several steps: attachment and membrane fusion, uncoating of the viral genome, translation of open-reading frames (ORFs) and non-structural proteins (NSPs), formation of double-membrane vesicles (DMVs), genome transcription and replication, and virion release (Fung and Liu, 2019). Once the virus enters the host, the two polyprotein precursors, ppla and pplab, are directly translated (Heer et al., 2020). Then, at least 16 NSPs are produced due to the serial cleavage of ppla and pplab (Hufsky et al., 2021). NSPs generally are localized at the endoplasmic reticulum (ER) to establish the DMVs for viral replication (Benvenuto et al., 2020). DMVs located in the perinuclear region of the host cells provide a microenvironment that promotes viral genome replication and protects the virion from clearance (Gui et al., 2019).

Autophagy is a conserved cellular process for maintaining cell homeostasis and eliminating misfolded proteins, damaged organelles, and pathogens (Yue et al., 2018). The autophagy signaling pathway consists of three steps: the formation of the initiation complex, the development of mature autophagosomes, and fusion with the lysosome (Fung and Liu, 2019; Ghosh et al., 2020). Viruses could lead to the generation of an autophagic response to viral infection. For most viruses, knocking out the autophagy-related genes (ATGs) would increase viral titers (Ismayil et al., 2020; Lal et al., 2020). Generally, double-stranded DNA (dsRNA) viruses activate the tripartite motif protein 32 (TRIM32)/tax1-binding protein 1 complex through TIR domaincontaining adapter molecule 1 (TRIF). Then, TRIF induces the autophagy-lysosome pathway to digest the virus by inhibiting Bcl-2 (Zhang et al., 2019). However, CoVs target different steps of the autophagy-lysosome pathway during infection (Zhao et al., 2021). Here, we suggest two main purposes for CoV autophagy evasion: the formation of protective structure DMVs and the suppression of autophagy flux by blocking the fusion of the autophagosome and the lysosome.

This minireview first summarizes the generation of DMVs, particularly the mechanism of $\mathrm{CoV}$-induced rearrangement of the cellular membrane. Next, we suggest the role of NSPs and ORFs in suppressing autophagy-lysosome fusion and cargo degradation. This minireview provides a comprehensive understanding of the mechanisms by which CoVs evade the autophagy-lysosome pathway. We hope it can contribute to discovering new drug targets and therapeutic strategies for COVID-19 in the future.

\section{CORONAVIRUSES HIJACK AUTOPHAGY INITIATION AND ERAD MACHINERY TO FORM DOUBLE-MEMBRANE VESICLES BY INDUCING MEMBRANE REARRANGEMENT}

The autophagy-lysosome pathway serves as the first line of defense against viral genome replication and expression but also as a potential source of host membrane (Vabret et al., 2020). The production of $\mathrm{CoV}$ particles is positively correlated with the number of DMVs (Maity and Saha, 2021; Miao et al., 2021). In recent years, the formation and development of DMVs have attracted enormous attention due to the similarities between DMVs and autophagosomes. However, the relationship between DMVs and the autophagy pathway has been controversial. Recent studies have revealed that inhibition of autophagy blocks viral replication, which implies that the autophagic structure is required for viral replication (Olzmann and Carvalho, 2019). However, according to accumulated studies, the generation of DMVs does not depend on autophagy. Based on existing reports, we suggest that autophagy-derived DMVs and ERderived DMVs are two different important pathways of DMV production. On the one hand, CoVs can hijack autophagy initiation to form unique DMVs. On the other hand, CoVs induce ER-derived DMVs independent of the autophagic pathway by hijacking host cell ER-associated degradation (ERAD) machinery (Konno et al., 2020).

According to recent reports, the NSP3 and NSP6 proteins of SARS-CoV-2 and MERS-CoV induce the formation of omegasome intermediates resident to the ER to form autophagyspecific phagophores, which results in the activation of the autophagy-lysosome pathway (Mihelc et al., 2021). Moreover, $\mathrm{N}$ protein and ORF8 of all three CoVs can activate the ULK1 complex to promote omegasome formation via the AMPK/mTOR signaling pathway (Snijder et al., 2020). SARSCoV ORF8b can enhance autophagy initiation by inducing the ER stress signaling pathway (Yang and Shen, 2020). DMVs mimic autophagosomes in structure. Non-lipidated LC3-I is associated with the outer membrane of the DMVs. However, the autophagosome-like DMVs do not contain LC3-II and lose the ability to fuse with lysosomes (Liu et al., 2019; VeraOtaroola et al., 2020). It has been reported that ORF3a of SARS-CoV-2 hijacks the lipidation of LC3, which inhibits the phosphatidylethanolamine (PE)-dependent conversion of LC3I to LC3-ll (Zou et al., 2019). The reduction of LC3-ll leads to the inhibition of the conversion of nucleating phagophores to autophagosomes. Additionally, NSP6 and the PLP2 domain of NSP3 of SARS-CoV and MERS-CoV promote the degradation of Beclin1 through the activation of SKP2 via the AKT signaling pathway, which inhibits vesicle nucleation and autophagosome formation (Gassen et al., 2019). Therefore, these non-nucleation phagophores and omegasomes self-use to autophagy-derived 
DMVs. CoVs can utilize autophagy-derived DMVs to protect their DNA and proteins from being cleared by lysosomes, which provides a venue for $\mathrm{CoV}$ replication.

Typical autophagosomes originate from the ER-Golgi intermediate complex (ERGIC) and consist of various ER/Golgimaker proteins, such as EDEM1, COP-ll, and RPN1. Therefore, the ER-Golgi is another important way to produce DMVs independent of the autophagy pathway. There is substantial evidence that $\mathrm{CoVs}$ induce membrane rearrangement by hijacking the host cell ERAD machinery (Miller et al., 2020; $\mathrm{Qu}$ et al., 2021). NSPs regulate ER and Golgi apparatus rearrangement to form DMVs directly from the ER (Gassen et al., 2019). CoV infection induces the accumulation of OS-9, EDEM1, and ERAD in the DMVs (Li et al., 2019). SARS-CoV-2 NSP4 and NSP6 induce ER-derived DMVs independent of the autophagic pathway by hijacking the host cell ERAD machinery (Muscolino et al., 2020). In addition, prior studies indicate that SARS-CoV NSP2 and MERS-CoV NSP3 could induce extensive rearrangement of cellular autophagosome-like vesicles around the ER and the Golgi apparatus (Pradel et al., 2020). The majority of host cells undergo ER stress after $\mathrm{CoV}$ infection. ER stress may be another target of NSPs, and it may be used as a driving force to promote the generation of DMVs and autophagosomes (Fung and Liu, 2019; Jin et al., 2020). SARS-CoV-2 ORF3-a has been reported to associate with non-lipidated LC3-I by activating ER stress, which promotes multiple-lamellar membrane structure emergence from the ER membrane, and prevents the generation of single-membrane structures (Alsaadi et al., 2020). Moreover, NSP6,7 of SARS-CoV-2 and NSP5,6 of SARS-CoV have been reported to block the EDEMsomes fusion with the late endosomes and then induce the membrane rearrangement to produce ER-derived DMVs (Maity and Saha, 2021; Tiwari et al., 2021). Therefore, membrane rearrangement by hijacking ERAD machinery to form ER-derived DMVs independently is another important way for viral replication.

Here we draw a mechanism model that describes how CoVs promote the generation of DMVs by inducing membrane rearrangement by different routines. First, CoVs hijack autophagy initiation and usurp the autophagy-lysosome pathway to form autophagy-derived DMVs. Second, CoVs induce ER-derived DMVs independently by hijacking ERAD machinery (Figure 1).

\section{CORONAVIRUSES USURP HOST CLEARANCE BY INTERCEPTING AUTOPHAGY FLUX}

The autophagy-lysosome pathway is a part of innate immunity, the first-line defense for eliminating pathogens. As part of the cellular defense system, autophagy protects against bacterial or viral infection by forming autophagosomes that encapsulate pathogens and transporting these cargoes to lysosomes for degradation (Zhao et al., 2021). Although CoVs hijack different steps of autophagy to promote DMV formation, there are still many autophagosomes produced in which viral RNA and proteins are enveloped and sent to the lysosomes for clearance
(Vabret et al., 2020). Latest reports indicate that when autophagy initiation was induced during $\mathrm{CoV}$ infection, the autophagy flux surprisingly was blocked (Krafcikova et al., 2020; Miao et al., 2021). Studies report that failure of lysosomal fusion with autophagosomes has been observed. Therefore, CoVs can escape the clearance of the autophagy-lysosome pathway by intercepting autophagy, a crucial component of $\mathrm{CoV}$ replication and survival.

A recent study shows that the expression of lysosomal membrane genes and lysosomal acidification genes is downregulated in Vero cells infected with CoVs (Gassen et al., 2019). Moreover, lysosomal enzymes are inactivated, and changes in lysosomal acidity have been observed in CoVinfected Vero cells (Ghosh et al., 2020). Further studies were performed to elucidate the underlying molecular mechanism. According to a recent study, ORF7a and ORF8b could directly regulate the $\mathrm{pH}$ of lysosomes in SARS-CoV-infected Hela cells (Hayn et al., 2021). ORF5 and ORF4b of MERS-CoV could downregulate the expression of the lysosomal membrane and lysosomal acidification genes (Singh et al., 2021). Previous studies suggested that SARS-CoV-2 NSP6 and ORF1a in all three CoVs could reduce the autophagosome-lysosome fusion (Hayn et al., 2021). Moreover, recent studies reveal the mechanism by which SARS-CoV-2 ORF3a blocks autophagosome maturation and the fusion of the autophagosome with lysosome by inhibiting the assembly of VAMP8 (a significant vesicle adaptor protein) complex through the regulation of a membrane fusion accelerator (such as HOPS, VPS39, and STX17) (Miao et al., 2021). In addition, MERS-CoV NSP6 can regulate Beclin1 ubiquitination, an important protein for autophagosomelysosome fusion (Wang et al., 2020). Moreover, the PLP-TM domain of NSP3 in all three CoVs binds Beclin1 and STING1 (stimulator of interferon response cGAMP interactor 1), which prevents Beclin1 from promoting autophagosome-lysosome fusion and inhibits the production of interferon (Zhang et al., 2021). Autophagy plays a pivotal role in IFN-I production (Zhu and Zheng, 2020). The NSP3 PLP-TM domain is necessary to complex Beclin1 to STING1, which prevents the stimulation of IFN production (Zhang et al., 2021). In the absence of IFNs, it is difficult for host cells to eliminate the virus through innate immunity (Pradel et al., 2020).

Therefore, there are two main purposes of CoVs intercepting autophagy flux. Firstly, CoVs usurp lysosome degradation by modulating lysosomal acidity or preventing autophagosome and lysosome fusion, which means CoVs evade the host first-line defense and protect viral DNA and proteins from being cleared (Figure 1). Secondly, the autophagy-lysosome pathway plays a pivotal role in IFN-I production as part of innate immunity. Blocking the autophagy flux is also important for CoVs usurping innate immunity elimination.

\section{DISCUSSION}

As a ssRNA virus, CoVs have spread globally, posing a serious threat to global public health. Major CoVs include SARS-CoV, MERS-CoV, and SARS-CoV-2. In most cases, CoVs possess the ability to usurp host clearance (Fung and Liu, 2019). Therefore, 




it is urgent and important to study the regulatory mechanism of virus evasion. Although CoVs evade the autophagylysosome pathway and innate immunity has been reported, many molecular mechanisms remain unclear, and many studies remain controversial (Pan et al., 2020). Many investigations have been conducted to determine the origin of DMVs and if autophagosomes are required for viral replication. This minireview aimed to elaborate on the relationship between DMVs and autophagosomes and the relationship between viral replication and autophagy. We also summarized the source of DMVs and how CoVs evade the autophagy-lysosome pathway (Figure 1).

Double-membrane vesicles protect virial DNA and proteins from being eliminated by lysosomes. The production of virus particles is positively correlated with the number of DMVs. Previously, there has been no distinction between autophagosomes and DMVs. Although DMVs and autophagosomes are similar in structure and co-localize with autophagy-related proteins, they differ in size, structure, and function (Nguyen et al., 2020). In this review, we summarize the source of DMVs during CoV infection and suggest that DMVs can form passively in an atypical autophagy process. CoVs hijack autophagy initiation through NSPs and ORFs, which leads to non-nucleation phagophores and omegasomes to autophagy-derived DMVs.

Additionally, membrane rearrangement by hijacking ERAD machinery to form ER-derived DMVs independent from the autophagy process is another important routine for DMV production. Existing research also suggests that DMVs are not the only venues for $\mathrm{CoV}$ replication. Recent studies suggest that autophagosomes are also the sites where the virus can replicate, and autophagosomes that do not fuse with lysosomes can also rupture and release virus particles (Hui et al., 2021). This view is consistent with CoVs preventing the fusion of autophagosomes and lysosomes. Autophagosomes belong to a specific DMV. Therefore, virus particles are derived from DMV and autophagosomes. Here we also conclude that CoVs usurp host clearance via intercepting autophagy flux. CoVs usurp lysosome degradation by modulating lysosomal acidity or preventing autophagosome and lysosome fusion. 
SARS-CoV-2-infected patients should not suppress the autophagy-lysosome pathway to clinical treatment, which may not inhibit the replication of the virus. In a randomized clinical trial, although limited by early termination, there was no clinical benefit of hydroxychloroquine administered daily for 8 weeks as pre-exposure prophylaxis in hospital-based HCWs exposed to patients with COVID-19 (Abella et al., 2021). It has been proven that autophagic flux inhibitors such as hydroxychloroquine (HCQ) may not be effective drugs, but autophagic inducers may prove better alternatives (Abella et al., 2021; Devarajan and Vaseghi, 2021). Autophagy is the negative regulation of innate immunity, which could prevent the development of host cellular inflammation (Liu et al., 2019; Vera-Otaroola et al., 2020). The NSP3 PLP-TM domain is necessary to complex Beclin1 to STING1, which prevents the stimulation of IFN production (Zhang et al., 2021). However, CoVs escaping the innate immunity whether dependent on hijacking the autophagylysosome pathway is still unclear. Meanwhile, a molecular mechanism of the transportation and location of viral proteins is still mysterious.

Currently, various countries and regions have begun vaccination, from institutions such as Pfizer, Oxford University, and Sinovac Biotech. As of December 2021, more than $54 \%$ of the people have been vaccinated (Devarajan and

\section{REFERENCES}

Abella, B. S., Jolkovsky, E. L., Biney, B. T., Uspal, J. E., Hyman, M. C., Frank, I., et al. (2021). Efficacy and safety of hydroxychloroquine vs placebo for pre-exposure SARS-CoV-2 prophylaxis among health care workers: a randomized clinical trial. JAMA Intern. Med. 181, 195-202. doi: 10.1001/jamainternmed.2020.6319

Alsaadi, E. A. J., Neuman, B. W., and Jones, I. M. (2020). Identification of a membrane binding peptide in the envelope protein of MHV coronavirus. Virus. Basel 12:1054. doi: 10.3390/v12091054

Benvenuto, D., Angeletti, S., Giovanetti, M., Bianchi, M., Pascarella, S., Cauda, R., et al. (2020). Evolutionary analysis of SARS-CoV-2: how mutation of nonstructural protein 6 (NSP6) could affect viral autophagy. J. Infect. 81, E24-E27. doi: 10.1016/j.jinf.2020.03.058

de Wit, E., Feldmann, F., Cronin, J., Jordan, R., Okumura, A., Thomas, T., et al. (2020). Prophylactic and therapeutic remdesivir (GS-5734) treatment in the rhesus macaque model of MERS-CoV infection. Proc. Natl. Acad. Sci. U.S.A 117, 6771-6776. doi: 10.1073/pnas.1922083117

Devarajan, A., and Vaseghi, M. (2021). Hydroxychloroquine can potentially interfere with immune function in COVID-19 patients: mechanisms and insights. Redox Biol. 38:101810. doi: 10.1016/j.redox.2020.101810

Fung, T. S., and Liu, D. X. (2019). Human coronavirus: host-pathogen interaction. Annu. Rev. Microbiol. 73, 529-557. doi: 10.1146/annurev-micro-020518115759

Gassen, N. C., Niemeyer, D., Muth, D., Corman, V. M., Martinelli, S., Gassen, A., et al. (2019). SKP2 attenuates autophagy through beclin1-ubiquitination and its inhibition reduces MERS-coronavirus infection. Nat. Commun. 10:5770. doi: 10.1038/s41467-019-13659-4

Ghosh, S., Dellibovi-Ragheb, T. A., Kerviel, A., Pak, E., Qiu, Q., Fisher, M., et al. (2020). Beta-coronaviruses use lysosomes for egress instead of the biosynthetic secretory pathway. Cell 183, 1520-1535. doi: 10.1016/j.cell.2020.10.039

Gui, X., Yang, H., Li, T., Tan, X. J., Shi, P. Q., Li, M. H., et al. (2019). Autophagy induction via STING trafficking is a primordial function of the cGAS pathway. Nature 567, 262-266. doi: 10.1038/s41586-019-1006-9

Hayn, M., Hirschenberger, M., Koepke, L., Nchioua, R., Straub, J. H., Klute, S., et al. (2021). Systematic functional analysis of SARS-CoV-2 proteins uncovers viral innate immune antagonists and remaining vulnerabilities. Cell Rep. 35:109126. doi: 10.1016/j.celrep.2021.109126
Vaseghi, 2021). However, there are still no effective targeted drugs currently. Therefore, developing new drug targets and a new therapeutic strategy is urgent. Our review details which coronavirus NSPs and ORFs promote DMV formation, how CoVs evade autophagy-lysosome degradation and innate immunity, and how this may contribute to developing novel therapies and clinical drugs against COVID19 in the future.

\section{AUTHOR CONTRIBUTIONS}

HWL and SL: conception and design and writing. HL and DL: revision of the manuscript. All authors read and approved the final manuscript.

\section{FUNDING}

This work was funded by the National Natural Science Foundation of China (No. 82002909), Chengdu Medical College Research Foundation (KYPY-20-01), and the Disciplinary Construction Innovation Team Foundation of Chengdu Medical College (CMC-XK-2101).

Heer, C. D., Sanderson, D. J., Voth, L. S., Alhammad, Y. M. O., Schmidt, M. S., Trammell, S. A. J., et al. (2020). Coronavirus infection and PARP expression dysregulate the NAD metabolome: an actionable component of innate immunity. J. Biol. Chem. 295, 17986-17996. doi: 10.1074/jbc.RA120. 015138

Hufsky, F., Lamkiewicz, K., Almeida, A., Aouacheria, A., Arighi, C., Bateman, A., et al. (2021). Computational strategies to combat COVID-19: useful tools to accelerate SARS-CoV-2 and coronavirus research. Brief. Bioinform. 22, 642663. doi: 10.1093/bib/bbaa232

Hui, X. F., Zhang, L. L., Cao, L., Huang, K., Zhao, Y., Zhang, Y. F., et al. (2021). SARS-CoV-2 promote autophagy to suppress type I interferon response. Signal Transduct. Target. Ther. 6:180. doi: 10.1038/s41392-021-00574-8

Ismayil, A., Yang, M., and Liu, Y. (2020). Role of autophagy during plant-virus interactions. Semin. Cell Dev. Biol. 101, 36-40. doi: 10.1016/j.semcdb.2019.07. 001

Jin, Z. M., Du, X. Y., Xu, Y. C., Deng, Y. Q., Liu, M. Q., Zhao, Y., et al. (2020). Structure of M-pro from SARS-CoV-2 and discovery of its inhibitors. Nature 582, 289-293.

Konno, Y., Kimura, I., Uriu, K., Fukushi, M., Irie, T., Koyanagi, Y., et al. (2020). SARS-CoV-2 ORF3b is a potent interferon antagonist whose activity is increased by a naturally occurring elongation variant. Cell Rep. 32:108185. doi: 10.1016/j.celrep.2020.108185

Krafcikova, P., Silhan, J., Nencka, R., and Boura, E. (2020). Structural analysis of the SARS-CoV-2 methyltransferase complex involved in RNA cap creation bound to sinefungin. Nat. Commun. 11:3717. doi: 10.1038/s41467-020-17 495-9

Lal, N. K., Thanasuwat, B., Chan, B., and Dinesh-Kumar, S. P. (2020). Pathogens manipulate host autophagy through injected effector proteins. Autophagy 16, 2301-2302. doi: 10.1080/15548627.2020.1831816

Li, S., Yuan, L., Dai, G., Chen, R. A., Liu, D. X., and Fung, T. S. (2019). Regulation of the ER stress response by the ion channel activity of the infectious bronchitis coronavirus envelope protein modulates virion release, apoptosis, viral fitness, and pathogenesis. Front. Microbiol. 10:3022. doi: 10.3389/fmicb.2019.0 3022

Liu, D., Wu, H., Wang, C. G., Li, Y. J., Tian, H. B., Siraj, S., et al. (2019). STING directly activates autophagy to tune the innate immune response. Cell Death Differ. 26, 1735-1749. doi: 10.1038/s41418-018-0251-z 
Maity, S., and Saha, A. (2021). Therapeutic potential of exploiting autophagy cascade against coronavirus infection. Front. Microbiol. 12:675419. doi: 10. 3389/fmicb.2021.675419

Miao, G., Zhao, H., Li, Y., Ji, M., Chen, Y., Shi, Y., et al. (2021). ORF3a of the COVID-19 virus SARS-CoV-2 blocks HOPS complex-mediated assembly of the SNARE complex required for autolysosome formation. Dev. Cell 56, 427-442.e5 doi: 10.1016/j.devcel.2020.12.010

Mihelc, E. M., Baker, S. C., and Lanman, J. K. (2021). Coronavirus infection induces progressive restructuring of the endoplasmic reticulum involving the formation and degradation of double membrane vesicles. Virology 556, 9-22. doi: 10.1016/j.virol.2020.12.007

Miller, K., McGrath, M. E., Hu, Z., Ariannejad, S., Weston, S., Frieman, M., et al. (2020). Coronavirus interactions with the cellular autophagy machinery. Autophagy 16, 2131-2139. doi: 10.1080/15548627.2020.1817280

Muscolino, E., Schmitz, R., Loroch, S., Caragliano, E., Schneider, C., Rizzato, M., et al. (2020). Herpesviruses induce aggregation and selective autophagy of host signalling proteins NEMO and RIPK1 as an immune-evasion mechanism. Nat. Microbiol. 5, 331-342. doi: 10.1038/s41564-019-0624-1

Nguyen, C. C., Domma, A. J., Zhang, H., and Kamil, J. P. (2020). Endoplasmic reticulum (ER) reorganization and intracellular retention of CD58 are functionally independent properties of the human cytomegalovirus ER-resident glycoprotein UL148. J. Virol. 94:e01435-19. doi: 10.1128/JVI.01435-19

Olzmann, J. A., and Carvalho, P. (2019). Dynamics and functions of lipid droplets. Nat. Rev. Mol. Cell Biol. 20, 137-155. doi: 10.1038/s41580-018-0085-z

Pan, H., Peto, R., Karim, Q. A., Alejandria, M., Henao-Restrepo, A. M., García, C. H., et al. (2020). Repurposed antiviral drugs for COVID-19 -interim WHO SOLIDARITY trial results. medRxiv [Preprint] doi: 10.1101/2020.10.15. 20209817

Pradel, B., Robert-Hebmann, V., and Espert, L. (2020). Regulation of innate immune responses by autophagy: a goldmine for viruses. Front. Immunol. 11:578038. doi: 10.3389/fimmu.2020.578038

Qu, J., Li, M., Li, D., Xin, Y., Li, J., Lei, S., et al. (2021). Stimulation of sigma1 receptor protects against cardiac fibrosis by alleviating IRE1 pathway and autophagy impairment. Oxid. Med. Cell Longev. 2021:8836818. doi: 10.1155/ 2021/8836818

Singh, K., Chen, Y. C., Hassanzadeh, S., Han, K., Judy, J. T., Seifuddin, F., et al. (2021). Network analysis and transcriptome profiling identify autophagic and mitochondrial dysfunctions in SARS-CoV-2 Infection. Front. Genet. 12:599261.

Snijder, E. J., Limpens, R., de Wilde, A. H., de Jong, A. W. M., Zevenhoven-Dobbe, J. C., Maier, H. J., et al. (2020). A unifying structural and functional model of the coronavirus replication organelle: tracking down RNA synthesis. PLoS Biol. 18:e3000715. doi: 10.1371/journal.pbio.3000715

Tiwari, R., Mishra, A. R., Gupta, A., and Nayak, D. (2021). Structural similaritybased prediction of host factors associated with SARS-CoV-2 infection and pathogenesis. J. Biomol. Struct. Dyn. 1-12. doi: 10.1080/07391102.2021.1874532

Vabret, N., Britton, G. J., Gruber, C., Hegde, S., Kim, J., Kuksin, M., et al. (2020). Immunology of COVID-19: current state of the science. Immunity 52, 910-941. doi: 10.1016/j.immuni.2020.05.002
Vera-Otaroola, J., Solis, L., Lowy, F., Olguin, V., Angulo, J., Pino, K., et al. (2020). The andes Orthohantavirus NSs Protein antagonizes the type I interferon response by inhibiting MAVS signaling. J. Virol. 94:e00454-20. doi: 10.1128/JVI. 00454-20

Wang, W. B., Hu, D. W., Wu, C. F., Feng, Y. Q., Li, A. X., Liu, W. Y., et al. (2020). STING promotes NLRP3 localization in ER and facilitates NLRP3 deubiquitination to activate the inflammasome upon HSV-1 infection. PLoS Pathog. 16:e1008335. doi: 10.1371/journal.ppat.1008335

Yang, N., and Shen, H. M. (2020). Targeting the endocytic pathway and autophagy process as a novel therapeutic strategy in COVID-19. Int. J. Biol. Sci. 16, 1724-1731. doi: 10.7150/ijbs.45498

Yue, Y., Nabar, N. R., Shi, C. S., Kamenyeva, O., Xiao, X., Hwang, I. Y., et al. (2018). SARS-coronavirus open reading frame-3a drives multimodal necrotic cell death. Cell Death Dis. 9:904. doi: 10.1038/s41419-018-0917-y

Zhang, G., Luk, B. T., Weil, X. L., Campbell, G. R., Fang, R. H., Zhang, L. F., et al. (2019). Selective cell death of latently HIV-infected CD $4^{+}$T cells mediated by autosis inducing nanopeptides. Cell Death Dis. 10:419. doi: 10.1038/s41419019-1661-7

Zhang, Y. B., Sun, H., Pei, R. J., Mao, B. L., Zhao, Z. Y., Li, H. H., et al. (2021). The SARS-CoV-2 protein ORF3a inhibits fusion of autophagosomes with lysosomes. Cell Discov. 7:31. doi: 10.1038/s41421-021-00268-Z

Zhao, Z., Lu, K., Mao, B., Liu, S., Trilling, M., Huang, A., et al. (2021). The interplay between emerging human coronavirus infections and autophagy. Emerg. Microbes Infect. 10, 196-205. doi: 10.1080/22221751.2021.1872353

Zhu, H. F., and Zheng, C. F. (2020). The race between host antiviral innate immunity and the immune evasion strategies of herpes simplex virus 1 . Microbiol. Mol. Biol. Rev 84:e00099-20. doi: 10.1128/MMBR.00099-20

Zou, D. H., Xu, J. X., Duan, X. L., Xu, X., Li, P. F., Cheng, L. X., et al. (2019). Porcine epidemic diarrhea virus ORF3 protein causes endoplasmic reticulum stress to facilitate autophagy. Vet. Microbiol. 235, 209-219. doi: 10.1016/j.vetmic.2019. 07.005

Conflict of Interest: The authors declare that the research was conducted in the absence of any commercial or financial relationships that could be construed as a potential conflict of interest.

Publisher's Note: All claims expressed in this article are solely those of the authors and do not necessarily represent those of their affiliated organizations, or those of the publisher, the editors and the reviewers. Any product that may be evaluated in this article, or claim that may be made by its manufacturer, is not guaranteed or endorsed by the publisher.

Copyright (C) 2022 Liang, Luo, Liao and Li. This is an open-access article distributed under the terms of the Creative Commons Attribution License (CC BY). The use, distribution or reproduction in other forums is permitted, provided the original author(s) and the copyright owner(s) are credited and that the original publication in this journal is cited, in accordance with accepted academic practice. No use, distribution or reproduction is permitted which does not comply with these terms. 